BBA 45949

\title{
FLUORESCENCE CHARACTERISTICS OF LYOPHILIZED MAIZE CHLOROPLASTS SUSPENDED IN BUFFER
}

\author{
RAJNI GOVINDJEE*, GOVINDJEE, J. LAVOREL AND J. M. BRIANTAIS \\ Department of Botany. University of Illinois, Urbana, Ill. 6I 80 I (U.S.A.) and Laboratoire de Photo- \\ synthèse, C.N.R.S., Gif-sur-Yvette (France)
}

(Received November 24th, 1969)

(Revised manuscript received February 27th, 1970)

\section{SUMMARY}

Fluorescence transients were measured in lyophilized maize chloroplasts (suspended in Tris-maleate buffer ( $\mathrm{pH} \mathrm{6.6)}$ ) after extraction with heptane. (The fluorescence characteristics before extraction were qualitatively similar to those in the fresh chloroplasts.) The initial fluorescence level $(m)$ in the (dry) heptane-extracted sample remained the same as in the unextracted material, but the variable fluorescence $(\Delta m)$ was drastically diminished. A portion of variable fluorescence, however, could be restored by adding $\mathrm{Na}_{2} \mathrm{~S}_{2} \mathrm{O}_{4}$. If the heptane extraction was made in the presence of water (wet), the $m$ level was almost as high as (or higher than) the final level $(M)$ of the unextracted sample, and $\Delta m$ was reduced. The "jet" of $\mathrm{O}_{2}$ (that measures the pool size of the intersystem intermediate A) and the "microjet" (that measures the pool size of the reaction center complex E), present in the unextracted samples, were absent in both types of extracted samples. Some of the above data may be interpreted in a hypothesis in which two quenchers $\left(Q_{1}\right.$ and $\left.Q_{2}\right)$ control the fluorescence $(O \rightarrow P)$ of chloroplasts - the reduction of $Q_{1}$ being responsible for the rapid and that of $Q_{2}$ for the slow fluorescence rise.

\section{INTRODUCTION}

LYNCH AND FRENCH ${ }^{1}$ and BISHOP ${ }^{2}$ showed that the light petroleum (b.p. 30-40 extraction of lyophilized chloroplasts destroys the Hill activity, but the latter could be restored by adding back the extract. BIsHop ${ }^{2}$ showed that the loss in activity was due to vitamin $\mathrm{K}$ (a quinone).

Chloroplasts contain several quinones ${ }^{3}$. There is I plastoquinone $\mathrm{A}(\mathrm{PQ}-\mathrm{A})$ per 7 chlorophyll molecules and I plastoquinone $\mathrm{C}(\mathrm{PQ}-\mathrm{C})$ for every 30 chlorophyll molecules". The compound " $Q$ ", the electron acceptor for Pigment System II, is, however, present in smaller quantities ( $\mathrm{r} / \mathrm{I} 50$ chlorophyll (Chl, ref. 5 ). There is a

\footnotetext{
Abbreviations: CMU, 3-(p-chlorophenyl)-I, I'-dimethylurea; DCMU, 3-(3,4-dichlorophenyl)-I, I-dimethylurea; Chl, chlorophyll; $\mathrm{PQ}$, plastoquinone.

* Present address: Department of Physiology and Biophysics, University of Illinois, Urbana, I11. 6r 80r, U.S.A.
} 
possibility that a special fraction of quinones serves as the component Q. AMESz AND FORK ${ }^{6}$ have shown that $70 \mu \mathrm{M}$ of certain oxidized quinones can quench $50 \%$ of the initial fluorescence rise and $15 \mu \mathrm{M}$ the variable fluorescence rise. They have further demonstrated that this quenching was not due to the stimulation of the electron transport because it occurred even in the presence of 3-(3,4-dichlorophenyl)I, I-dimethylurea (DCMU). However, no definite evidence exists regarding the nature of $Q$.

$\mathrm{PQ}-\mathrm{C}$ is a more likely candidate to be " $Q$ " because of the closeness of its $E_{0}$ ' $(=55 \mathrm{mV})$, to that of $Q\left(E_{0}{ }^{\prime}=-35 \mathrm{mV}\right)^{7}$; the $E_{0}{ }^{\prime}$ of PQ-A is II $3 \mathrm{mV}$. TREBsT ${ }^{8}$ has shown that extraction of $70 \%$ of the total $\mathrm{PQ}-\mathrm{A}$ does not impair the $\mathrm{NADP}+$ reduction in chloroplasts and WoOD et al. ${ }^{9}$ have shown that PQ-A restores only about $30 \%$ of the Hill activity when added to the extracted chloroplasts. However, the crude extract restores about $70 \%$ of the activity. Thus, other factors are involved besides $P Q-A$. The above results, however, do not exclude the possibility that $Q$ is a special type of plastoquinone present in small quantities. Recently H. T. WITT (personal communication) has suggested that the primary acceptor of electrons of System II is a plastoquinone that is reduced first to a semiquinone.

In this paper we report the fluorescence characteristics of chloroplasts extracted with heptane, a universal solvent for quinones. Two methods of extraction were used. In one case, lyophilized chloroplasts were extracted with "dry" heptane. In the other case, lyophilized chloroplasts were extracted in the presence of a trace of water; this will be referred to as the "wet" heptane extraction. We present data on the time-course of fluorescence, the effects of a poison of photosynthesis 3 -( $p$-chlorophenyl)-I, $\mathrm{I}^{\prime \prime}$ dimethylurea (CMU), an electron acceptor $\left(\mathrm{Fe}(\mathrm{CN})_{6}{ }^{3-}\right)$ and a reductant $\left(\mathrm{Na}_{2} \mathrm{~S}_{2} \mathrm{O}_{4}\right)$ on the fluorescence transient, the fluorescence yield as a function of light intensity, the fluorescence spectra of constant and variable fluorescence and the rate of $\mathrm{O}_{2}$ exchange in these preparations.

To explain our results, we propose that two quenchers $\left(Q_{1}\right.$ responsible for rapid fluorescence changes and $Q_{2}$ for slow fluorescence changes) are involved in the $O \rightarrow P$ (ref. Io) rise. The wet heptane extraction causes extraction of $Q_{2}$. The dry heptane extraction destroys or extracts $Z H$ (the primary donor of System II) and keeps $Q_{2}$ unavailable for reduction. In both cases the bulk of the plastoquinones are extracted. The absence of $\mathrm{O}_{2}$ evolution suggests that in both cases some intermediate is inoperative. Attempts to reconstruct the original systems have been unsuccessful so far. Tentatively, we also entertain the hypothesis that a portion of $Q$ may not be a real substance - it may represent a state of the matrix surrounding Chl a.

\section{MATERIALS AND METHODS}

Zea mays (maize) was grown in the greenhouse for I month. $20 \mathrm{~g}$ of leaves were blended in Ioo $\mathrm{ml}$ of $0 . \mathrm{I} \mathrm{M}$ Tris-maleate, $0.4 \mathrm{M}$ sucrose buffer ( $\mathrm{pH} \mathrm{6.6)}$ (ref. II). The material was filtered through cheesecloth and cotton. The filtrate was centrifuged for $2 \mathrm{~min}$ at $200 \times \mathrm{g}$. The pellet was washed and suspended in $0 . \mathrm{I}$ M Tris-maleate buffer ( $\mathrm{pH}$ 6.6). The chloroplasts were then lyophilized.

For each experiment three equal quantities of lyophilized chloroplasts were weighed: (i) was suspended in o.I M Tris-maleate buffer ( $\mathrm{pH} \mathrm{6.6).} \mathrm{(ii)} \mathrm{was} \mathrm{extracted} \mathrm{with}$ heptane in a homogenizer for $\mathrm{I}_{2}-\mathrm{I} 5 \mathrm{~min}$ at room temperature and then centrifuged; 
the heptane was decanted and the chloroplasts dried under vacuum to remove the traces of heptane, and finally suspended in o.I $\mathrm{M}$ Tris-maleate buffer ( $\mathrm{pH}$ 6.6). (iii) was extracted with heptane (after being wet with a trace of water) for 12-15 min and then treated as in (ii).

The amount of chlorophyll per $\mathrm{ml}$ of the final suspension was adjusted to be IO-I $2 \mu \mathrm{g}$ Chl per $\mathrm{ml}$ suspension in all three samples.

Fluorescence measurements were made by the stop and flow method of $\mathrm{LA}^{-}$ VOREL ${ }^{12}$. When the suspension is flowing at a fast speed, the constant fluorescence $(m)$ is recorded; and when the flow is stopped, the fluorescence rises from $m$ to $M$ (the final level). In each case a record was made of the constant $(m)$ and the variable plus constant $(M)$ fluorescence, excited by $480-\mathrm{nm}$ light obtained from a monochromator. The light source was a Xenon lamp operated at $40 \mathrm{~A}$. Fluorescence was collected at right angles, passed through a Bausch and Lomb monochromator and detected by an EMI $9558 \mathrm{~A}$ photomultiplier.

$\mathrm{O}_{2}$ measurements were made on all samples by means of a platinum rate electrode. The chlorophyll concentration (in these measurements) was adjusted to approx. I $\mathrm{mg} \mathrm{Chl} \mathrm{per} \mathrm{ml} \mathrm{O}_{2}$ spike was measured by illuminating the chloroplasts for Io sec with $650-\mathrm{nm}$ light after 50-sec dark intervals. The microjet of $\mathrm{O}_{2}$ was'measured by using far-red light flashes of $10 \mu \mathrm{sec}$ given every $\mathrm{I} 5 \mathrm{sec}$.

RESULTS AND DISCUSSION

\section{(I) Fhorescence induction}

(A) Unextracted lyophilized chloroplasts suspended in buffer

Fluorescence induction in suspensions of fresh isolated chloroplasts ${ }^{13-19}$ is simpler than in whole cells (see literature citations in refs. 20 and 2r). A dark-adapted suspension of chloroplasts, when exposed to bright light (approx. Io ${ }^{15}$ quanta $\cdot \mathrm{cm}^{-2}$. $\left.\mathrm{sec}^{-1}\right)$ shows an instantaneous rise in fluorescence referred to here as minimum level $m\left(=F_{0}\right)$; if the yield of $m$ is independent of intensity, it is " $\mathrm{O}$ " (ref. Io). This is followed by a biphasic rise to a maximum level referred to here as $M$ level $\left(=F_{\infty}\right)$; when the yield of $M$ is truly maximum, it is " $\mathrm{P}$ " (ref. 10 ).

Fig. I shows a plot of the time $(t)$ it takes to obtain $(M-m) / 2$ (from the time the flow of the chloroplast suspension is stopped) as a function of light intensity in fresh and in lyophilized chloroplasts suspended in buffer. In all cases, this time $(t)$ increased with any decrease in intensity, but lyophilization did cause a slowing down of the rise from $m$ to $M$. This increase in $t$ was more pronounced when lyophilization was made by suspending chloroplasts in water rather than in buffer.

The kinetics of the rise ( $m$ to $M$ ) in lyophilized chloroplasts was similar to that in the fresh chloroplasts. First, there was a fast phase $(O \rightarrow I$, refs. 20-22); it was over within I sec. This phase was followed by a slow phase $(I \rightarrow P)$; at certain intensities (see refs. 5 and II) and also in the presence of certain concentrations of DCMU (or CMU) a plateau or a dip (D in ref. 2o) is observed between the fast and the slow phases. The slow rise is over within $5^{-15} \mathrm{sec}$ depending on the intensity of the exciting light and the physiological "history" of the chloroplasts. In chloroplasts, a decline from $M$, although extremely slow, is present and has often been called the steady state (S).

Intensity dependence. For the purpose of ensuring that the $m$ level was truly 

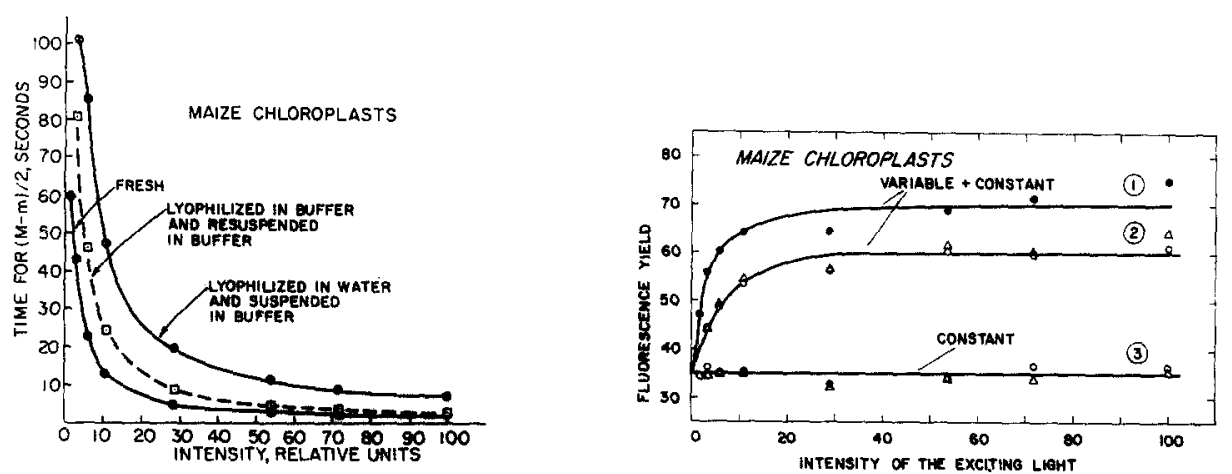

Fig. I. Time to reach half-maximal variable fluorescence $((M-m) / 2)$ as a function of the intensity of exciting light. ( 100 on the abscissa is equivalent to $10^{16} \mathrm{ergs} \cdot \mathrm{cm}^{-2} \cdot \mathrm{sec}^{-1}$.) Buffer: 0.1 M Trismaleate, $0.4 \mathrm{M}$ sucrose ( $\mathrm{pH} 6.6) ; 20^{\circ}$; $10 \mu \mathrm{g} \mathrm{Chl}$ per $\mathrm{ml}$ suspension of maize chloroplasts.

Fig. 2. Fluorescence yield $(F / I)$ for $m$ (constant) and $M$ (variable + constant) as a function of the intensity of exciting light. Curve I, $M$ for fresh chloroplasts in buffer; Curve $2, M$ for chloroplasts lyophilized in water $(\Delta)$ and in buffer $(O)$, resuspended in buffer; Curve $3, m$ for lyophilized chloroplasts - fresh chloroplasts had the same yield (points not shown). Note that no points are plotted for very low intensities. See legend of Fig. I.

constant fluorescence (O of LAVOREL $\left.{ }^{10}\right)$ and $M$ level was indeed variable plus constant fluorescence, we measured the induction of fluorescence as a function of light intensity and plotted the dependence of the yield of $M$ and $m$ on the intensity of exciting light. Fig. 2 shows a plot of $F / I$ versus $I$ for $m$ and $M$ levels in fresh chloroplasts and in lyophilized preparations. The Curve 3 shows that the fluorescence yield of $m$ (measured with a flowing system) in lyophilized and in fresh chloroplasts is indeed independent of light intensity. Thus, we must be monitoring true $O$. Curves $I$ and 2 are for $M$ of fresh and resuspended lyophilized chloroplasts. Both have the characteristics of the variable fluorescence (see refs. 20 and 23 for green plants and ref. 24 for photosynthetic bacteria) - the yield remains low and constant at low intensities, then rises and attains a high (about 2-3 times that at low intensity) and constant value. The ratio of $M$ to $m$ was about $\mathrm{r} .7$ for the lyophilized sample - quite close to that in fresh chloroplasts (2.0). The above results ensured that the lyophilization changed the system only slightly and quantitatively.

Effect of CMU. Lyophilized chloroplasts suspended in buffer containing Io $\mu \mathrm{M}$ CMU showed the same $m$ level as the untreated sample (Fig. 3, left curves); the fast rise from $m$ to $M$ was slightly accelerated (I6\% at $0.5 \mathrm{sec}$ after the flow was stopped), but the slow second phase of the rise from $m$ to $M$ was significantly decreased $(35 \%$, $4 \mathrm{sec}$ after the flow was stopped). At a lower intensity $(I=0.5)$, the increase in the first phase was higher $(37.5 \%$ at $0.5 \mathrm{sec})$ but the decrease at $4 \mathrm{sec}$ was still the same as at the higher intensity.

The differential effect of CMU on the two phases of the $m \rightarrow M$ rise is not consistent with the hypothesis that the CMU simply acts as a block between $Q$ and $A$ (ref. 25), in which case one might expect a more rapid $m \rightarrow M$ rise (as observed) but not a decrease in $M$. Another possibility would be that, in chloroplasts, another "factor" different from $Q$ controls the slow second phase of the $m \rightarrow M$ rise.

As a working hypothesis, we suggest that the chloroplast fluorescence is controlled by two quenchers $\left(Q_{1}\right.$ and $\left.Q_{2}\right)$. The reduction of $Q_{1}$ is responsible for the rapid 


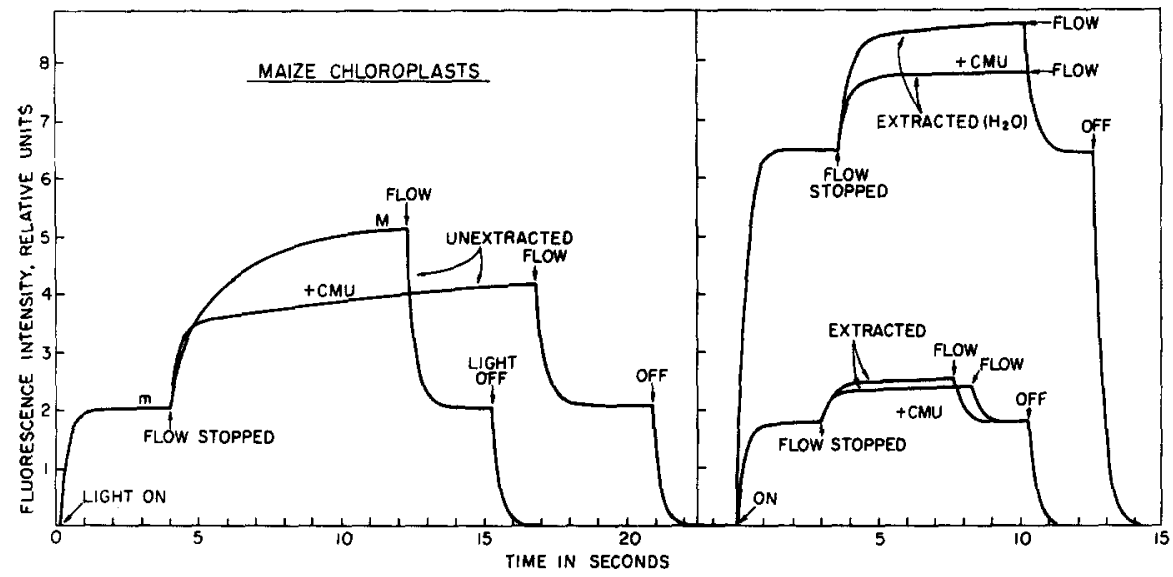

Fig. 3. Fluorescence induction in the lyophilized and resuspended chloroplasts with and without Io $\mu \mathrm{M}$ CMU. $m=$ constant fluorescence, $M=$ variable + constant fluorescence, as measured by the stop-flow method of LAvoreL. Left curves, unextracted chloroplasts; right and top curves, chloroplasts extracted with heptane (wet); right and bottom curves, chloroplasts extracted with heptane (dry). See legend of Fig. I.

rise and the reduction of $Q_{2}$ is responsible for the slow rise; both are quenchers in their oxidized forms but not in their reduced forms. CMU keeps the $Q_{2}$ in the oxidized form, abolishing the slow rise; however, the $Q_{1}$ to $Q_{1} \mathrm{H}$ reaction is accelerated because there is no competition with $Q_{2}$. (An explanation of the effect of DCMU on whole cells, however, meets with difficulty in this hypothesis.)

(B) Effect of extraction with dry heptane

The $m$ level of extracted chloroplasts (dry) remained essentially the same as it is in the unextracted sample (see bottom right curves, Fig. 3), but the variable fluorescence was drastically reduced. The ratio of $M$ to $m$ was 1.36 in contrast to 2.22 for the unextracted sample. In several other experiments, extraction led to still lower values (I.I and I.2) of $M / m$. Addition of Io $\mu \mathrm{M}$ CMU did not affect the $m$ level; the $m \rightarrow M$ rise, that was slightly slowed down in the extracted sample, was also unaffected, but the $M$ was decreased ( $24 \%, 4 \mathrm{sec}$ after the flow was stopped). Addition of an excess of $\mathrm{Na}_{2} \mathrm{~S}_{2} \mathrm{O}_{4}$ led to an increase of both $m$ and $M$ by about $25 \%$ (or more) suggesting that $Q_{1}$ was still present but could not be reduced in light. The complete variable fluorescence was not restored because dry heptane extraction keeps $Q_{2}$ in the oxidized form just as the CMU does. Reduction in the variable fluorescence has been reported when chloroplasts were heated or treated with ultraviolet light ${ }^{15,19}$. These results are consistent with the hypothesis that, in addition to other effects, $\mathrm{ZH}$ (the primary donor of electrons for System II) is destroyed with heat and ultraviolet light and extracted (or destroyed) with heptane. Yamashita AND BUTLER ${ }^{26}$ have shown that a block, created on the $\mathrm{ZH}$ side by suspending chloroplasts in $0.8 \mathrm{M}$ Tris, can be bypassed by adding reduced phenylenediamine which serves as an electron donor. When o.I mM phenylenediamine was added to the extracted chloroplasts (dry), the small "left-over" variable fluorescence was completely quenched without affecting the $m$ level. We raised the question: is it that the electron transfer is faster with phenylenediamine than without? We tested this by adding $50 \mu \mathrm{M} \mathrm{CMU}$ to the system - the reduction of the variable fluorescence was still present. Thus, the effect of o.I mM reduced phenylenediamine, in our system, is not due to an increased electron 
transfer. The reaction center complex may have been hampered by o.I mM phenylenediamine in our system.

(C) Effect of extraction with heptane in the presence of a trace of water

Lyophilized chloroplasts extracted with heptane (after the material was wetted with a trace of water) showed a very high constant fluorescence $(m)$ and a small variable fluorescence. The $m$ level in such preparations was equal to or higher than $M$ level of the unextracted samples (right top curves, Fig. 3). Table I shows the variability observed in several experiments: the variable fluorescence was reduced by

TABLE I

VARIATIONS IN THE EFFECT OF EXTRACTION IN THE PRESENCE OF WATER ON THE $m$ AND $M$ LEVELS

\begin{tabular}{|c|c|c|c|c|c|}
\hline $\begin{array}{l}\text { Expt. } \\
\text { No. }\end{array}$ & Conditions & $m$ & $\dot{M}$ & $\Delta m$ & $\Delta m / m$ \\
\hline \multirow[t]{2}{*}{ I } & Unextracted & . 8 & 22 & 14 & I. 8 \\
\hline & Extracted & I8 & 25 & 7 & 0.4 \\
\hline \multirow[t]{2}{*}{2} & Unextracted & I 5 & $3 I$ & 16 & I.I \\
\hline & Extracted & $3 I$ & 32 & $\mathbf{I}$ & 0.3 \\
\hline \multirow[t]{2}{*}{3} & Unextracted & I 8 & 35 & 17 & 0.95 \\
\hline & Extracted & 42 & $5 I$ & 9 & 0.2 \\
\hline \multirow[t]{3}{*}{4} & 'Unextracted & Io & 22 & I 2 & I. 2 \\
\hline & Extracted & $2 I$ & 24 & 3 & O. I 4 \\
\hline & $\therefore$ & & & & 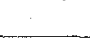 \\
\hline
\end{tabular}

$5^{0-95 \%}$ upon extraction and the $\Delta m / m$; that ranged from 0.95 to $\mathrm{I} .8$ in the unextracted samples, were 0.13 to 0.4 in the extracted samples. In all experiments (except Expt. 3, and that plotted in Fig. 3) the increase in $m$ upon extraction was approximately equal to the decrease in $\Delta m$. The variable fluorescence consisted of the fast component, the slow component being almost absent.

We suggest that the high $m$ level and the decreased $\Delta m$, is due to a partial extraction (or destruction) of $Q_{2}$; the $Q_{1}$ reaction is almost intact. The level $m$ in some experiments is higher than $M$ of the unextracted sample because the latter is not a true $P$, since $A$ is still present and does not allow a complete reduction of $Q$ to $Q H$. An alternative (or supplementary) explanation is to suggest that a change in the structure of the chloroplasts is partly responsible for the observed increase in $m$ (in particular in Expt. 3, Table I, and Fig. 3); this is a likely interpretation because the ratios of the emission spectra for the $\Delta m$ to $m$ level showed slight fluorescence due to $\mathrm{Chl} b$ and Chl $a 670$.

We tested if the (wet) extract had more PQ-A (if it is $Q_{2}$ ) than the (dry) extract by assaying this plastoquinone using silica-gel chromatography ${ }^{27}$. The amount of PQ-A was, however, found to be identical in the extracts obtained by the wet and dry methods of extraction. This may imply that $Q_{2}$ is not identical with PQ-A. (However, since $Q_{2}$ may be in small quantities, no definite conclusion can yet be made.)

Effect of $C M U$. The $m$ level and the fast phase of $m \rightarrow M$ rise was not affected by to $\mu \mathrm{M} \mathrm{CMU}$, but the $M$ level was slightly reduced $(38 \%, 4 \mathrm{sec}$ after the flow was stopped at $I=1.0$, and $26 \%$ when $I=0.5$ ). For this effect, we may suggest that the "left-over" $Q_{2}$ was now kept in the oxidized form as in the unextracted case. 
Effect of $\mathrm{Fe}(\mathrm{CN})_{6}{ }^{3-}$. MURATA et al. ${ }^{15}$ and MaLkIN AND KoK ${ }^{5}$ have shown that $\mathrm{Fe}(\mathrm{CN})_{6}{ }^{3-}$ decreases the slow variable fluorescence in chloroplasts because it acts as an electron acceptor and $\mathrm{QH}$ is not allowed to accumulate. We tested the effect of $\mathrm{Fe}(\mathrm{CN})_{\mathbf{6}}{ }^{3-}$ on our extracted samples; the variable, but not the constant, fluorescence decreased by $50 \%$.

Effect of reduced phenylenediamine. The addition of $60 \mu \mathrm{M}$ reduced phenylenediamine (in dark) - like CMU - reduced the slow variable fluorescence (Table II); this may be interpreted to mean that phenylenediamine affects the reaction center just like $\mathrm{CMU}$. Further addition of $\mathrm{CMU}$ or $\mathrm{Fe}(\mathrm{CN})_{6}{ }^{3-}$ does not cause any change. At a higher concentration of reduced phenylenediamine (o.I $\mathrm{mM}$ ), however, the variable fluorescence was totally absent.

TABLE II

EFFECT OF REDUCED PHENYLENEDIAMINE

\begin{tabular}{|c|c|c|c|c|c|}
\hline $\begin{array}{l}\text { Expt. } \\
\text { No. }\end{array}$ & Conditions & $m$ & $M$ & $\Delta m$ & $\Delta m / m$ \\
\hline I & $\begin{array}{l}\text { Extracted (in the presence of water) } \\
+60 \mu \mathrm{M} \text { reduced phenylenediamine (after I I min) } \\
+\mathrm{K}_{3} \mathrm{Fe}(\mathrm{CN})_{6}\end{array}$ & $\begin{array}{l}29 \\
31 \\
30\end{array}$ & $\begin{array}{l}44 \\
39 \\
39\end{array}$ & $\begin{array}{r}\text { I } 5 \\
8 \\
9\end{array}$ & $\begin{array}{l}0.52 \\
0.26 \\
0.30\end{array}$ \\
\hline 2 & $\begin{array}{l}\text { Extracted (in the presence of water) } \\
+ \text { o. I } \mathrm{mM} \text { reduced phenylenediamine } \\
+ \text { Io } \mu \mathrm{M} \text { CMU }\end{array}$ & $\begin{array}{l}27 \\
32 \\
32\end{array}$ & $\begin{array}{l}35 \\
32 \\
32\end{array}$ & $\begin{array}{l}8 \\
0 \\
0\end{array}$ & $\begin{array}{l}0.3 \\
0 \\
0\end{array}$ \\
\hline
\end{tabular}

Attempts to reconstitute the system. The heptane extract was readded to the sample, and the fluorescence induction reexamined. We did not succeed in obtaining the original induction (there was only a slight decrease in $m$, and the $\Delta m / m$ ratio slightly increased from 0.3 to 0.5 ). Perhaps, the heptane extraction caused other changes in the photosynthetic system that could not be restored, or else the extracted intermediates could not get to the right place during the reconstitution experiment.

The failure to achieve extraction of $Q_{1}$, the ability to "extract" $Q_{2}$ and only a small effect of CMU on the variable fluorescence in the extracted samples raises the question as to whether a portion (or all) of " $Q$ "' is really a substance. An alternative concept might be that a portion (or all) of $Q$ represents a state of matrix surrounding some special chlorophyll molecules ( $\mathrm{Chl} a_{\mathrm{II}}$ ) where a local trap would be available for an electron photoejected from the excited molecules. Such a trap localized in the vicinity of the special chlorophyll molecules would be functionally equivalent to the hypothetical substance $Q$. This situation seems to be well documented for dyes embedded in solid matrix at low temperatures ${ }^{28}$.

\section{(2) Emission spectra}

Fig. 4 shows the emission spectra for the constant $(m)$ and variable $(\Delta m)$ fluorescence of the unextracted maize chloroplasts; the two curves are arbitrarily adjusted at $660 \mathrm{~nm}$ and have been corrected for the transmission efficiency of the monochromator and the spectral sensitivity of the photomultiplier. Both have a peak at $682.5 \mathrm{~nm}$, but the emission spectrum of the variable fluorescence is different 
from that of the constant fluorescence: variable fluorescence is enriched in F685 (System II) relative to fluorescence at $700 \mathrm{~nm}$.

Fig. 5 shows the emission spectra of variable and constant fluorescence in lyophilized chloroplasts extracted with heptane in the presence of water. Here, the $697-\mathrm{nm}$ band in addition to the $682.5-\mathrm{nm}$ band is seen very clearly in the variable fluorescence. Similar results have recently been obtained by GovinDJEE AND LAVOREL
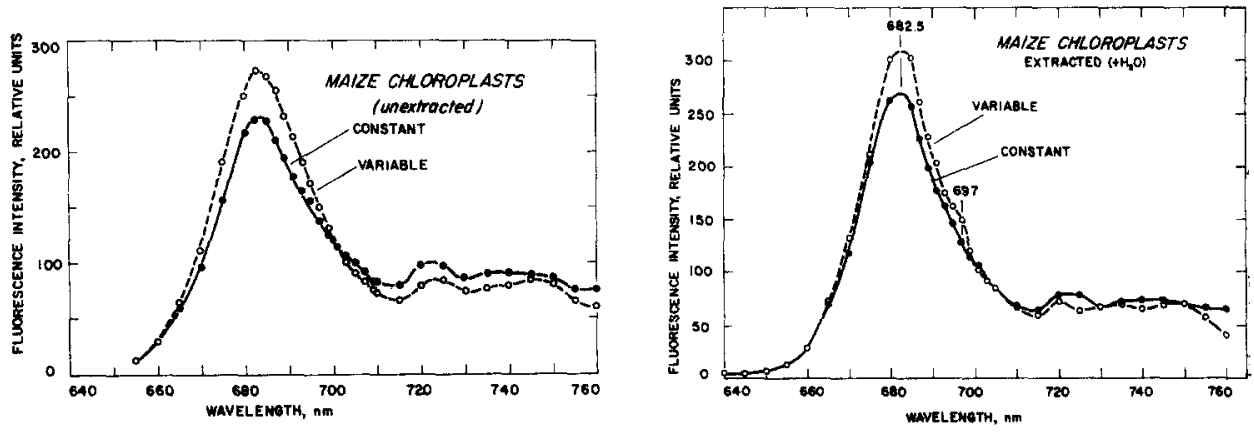

Fig. 4. Emission spectra of constant $(m)$ and variable $(\Delta m=M-m)$ fluorescence of unextracted lyophilized chloroplasts suspended in buffer. The curves were arbitrarily adjusted to give the same value at $660 \mathrm{~nm}$. See legends of Figs. I-3.

Fig. 5. Emission spectra of constant $(m)$ and variable $(\Delta m=M-m)$ fluorescence of lyophilized chloroplasts extracted in the presence of water (wet). See legends of Figs. I-4.

(unpublished) in Chlorella. GovinDJEE and co-workers ${ }^{28,30}$ observed a band at $693 \mathrm{~nm}$ in the emission spectrum of Porphyridium cruentum when excited with light of high intensity; they speculated that the $693-\mathrm{nm}$ band was due to the trap of System II. Perhaps the $69 \mathrm{I}-697-\mathrm{nm}$ bands are from similar complexes in chloroplasts from maize. (For the identification of the absorption bands with the emission bands, in vivo, see ref. 3I.)

A comparison of the emission spectra of $m$ extracted (dry) with that of the unextracted sample showed that the changes were negligible.

A comparison of the emission spectra of $\Delta m$ unextracted with that of the $\Delta m$ extracted (wet) showed a slightly larger proportion of $69 \mathrm{I}-697-\mathrm{nm}$ fluorescence in the extracted samples; it is to be noted that the $\Delta m$ extracted (wet) is mainly the fast component $(\mathrm{O} \rightarrow \mathrm{I})$, but $\Delta m$ unextracted includes the slow rise as well. The differences in the $\Delta m$ 's is a new thing; it means that the emission spectrum in the $\mathrm{O} \rightarrow \mathrm{I}$ phase may be different from that in the $I \rightarrow P$ phase.

\section{(3) $\mathrm{O}_{2}$ measurements}

The unextracted samples showed the presence of a prominent $\mathrm{O}_{2}$ spike when excited with $647-\mathrm{nm}$ light (Fig. 6). The addition of 7 ro-nm light led to an $\mathrm{O}_{2}$ uptake and the addition of $647-\mathrm{nm}$ light to 7 ro-nm light led to increased $\mathrm{O}_{2}$ evolution (Emerson effect). This confirms that the entire electron transport chain was intact in the lyophilized samples. These samples also showed the microjet of $\mathrm{O}_{2}$ with far-red light flashes of $10^{-5} \mathrm{sec}$ given every $\mathrm{I}_{5} \mathrm{sec}$. (The negative oxygen spike was also seen.) The extracted samples (both kinds) exhibited an extremely reduced $\mathrm{O}_{2}$ spike showing that some electron carrier(s) was missing; no microjet could be detected suggesting 
that the complex at the reaction center $\mathrm{ZH}$ Chl $a_{\text {II }} Q$ was affected. Examination of $\mathrm{Chl} a_{\mathrm{II}}$ absorbance changes showed ${ }^{32}$ that in the (dry) heptane-extracted chloroplasts these changes were absent, but they were distinctly present in the (wet) heptaneextracted chloroplasts and in the unextracted ones. It appears that the loss of $\mathrm{ZH}$ (but not that of $Q_{2}$ ) destroys the $\mathrm{Chl} a_{\Pi \mathrm{II}}$ changes; perhaps $\mathrm{ZH}$ and $Q_{1}$ are very tightly complexed with the active $\mathrm{Chl} a_{\mathrm{II}}$ molecules.

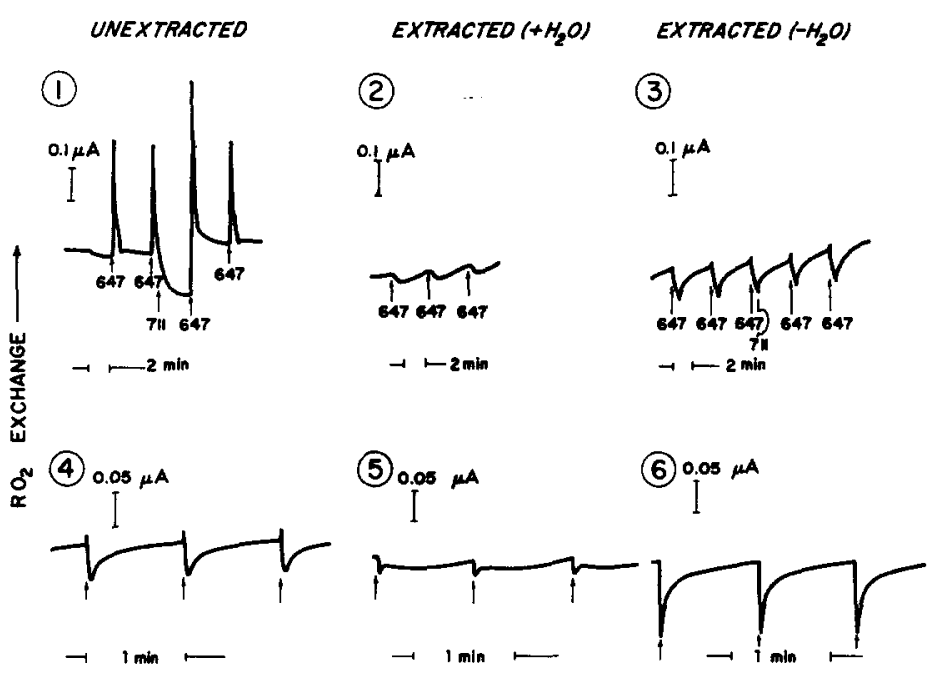

Fig. 6. Rate of $\mathrm{O}_{2}$ exchange (measured by a platinum rate electrode) in lyophilized chloroplasts suspended in buffer. Curves I-3, Io sec 647-nm light, and 50 sec dark (jet of $\mathrm{O}_{2}$ due to the pool of A); Curves 4-6, ro- $\mu$ sec far-red light flashes given every ${ }_{5} 5 \mathrm{sec}$ (microjet of $\mathrm{O}_{2}$ due to the pool of $\mathrm{E}$, and negative $\mathrm{O}_{2}$ spike). Left curves ( $\mathrm{I}$ and 4 ), unextracted chloroplasts; middle curves ( 2 and $5)$, extracted in the presence of water (wet); right curves ( 3 and 6 ), extracted in the absence of water (dry). I $\mathrm{mg} \mathrm{Chl}$ per $\mathrm{ml}$ suspension; $20^{\circ}$; air-saturated system.

\section{ACKNOWLEDGMENTS}

We thank Madam J. Maroc for her help in the assay of plastoquinones. The GovindJEEs are grateful to all members of the Laboratoire de Photosynthèse for their hospitality and cooperation. G. was supported by National Science Foundation GB 733I and GB I4I76. R.G. was supported by Laboratoire de Photosynthèse, C.N.R.S. J.M.B. was supported by the University of Illinois Research Board (1969-1970).

\section{REFERENCES}

I V. Lynch and C. S. French, Arch. Biochem. Biophys., 70 (1957) 382.

2 N. I. Bishop, Proc. Natl. Acad. Sci. U.S., 45 (1959) I 696

3 F. L. CRANe, in T. P. Singer, Biological Oxidations, Interscience, New York, 1968, p. 533.

4 M. D. Heninger and F. L. Crane, J. Biol. Chem., 242 (I967) I 55.

5 S. Malkin and B. KoK, Biochim. Biophys. Acta, I26 (1966) 413.

6 J. Amesz and D. C. Fork, Biochim. Biophys. Acta, 143 (1967) 97.

7 W. Cramer and W. Butler, Biochim. Biophys. Acta, I 72 (I969) 503.

8 A. Trebst, Proc. Roy. Soc. London, Ser. B, 157 (1963) 355. 
9 P. M. Wood, H. N. Bhagavan and F. L. Crane, Plant Physiol., 4 I (1966) 633.

IO J. LAVOREL, Plant Physiol., 34 (1959) 204.

I I Y. DE Kouchkovsky, Physiol. Végétale, I (1963) I5.

I J. Lavorel, Photochem. Photobiol., 4 (1965) 8 Ig.

I3 J. M. Briantais, Photochem. Photobiol., 5 (I966) 135.

I 4 Y. De Kouch Kovsky and P. Joliot, Photochem. Photobiol., 6 (1967) 567.

is N. Murata, M. Nishimura and T. Takamiya, Biochim. Biophys. Acta, i2o (I966) 23.

I6 B. Forbush and B. KoK, Biochim. Biophys. Acta, I62 (1968) 243.

I7 S. Malkin, Biochim. Biophys. Acta, r26 (I966) 433.

18 S. Malkin, Biochim. Biophys. Acta, I53 (r968) I 88.

Ig S. MaLkin and L. W. Jones, Biochim. Biophys. Acta, I62 (rg68) 297.

20 J, C. Munday, JR. And Govindjee, Biophys. J., 9 (1969) I.

2 I J. C. Munday, JR. And Govindjee, Biophys. J., 9 (I969) 2 I.

22 R. Delosme, Biochim. Biophys. Acta, I43 (1967) 108.

23 J. Lavorel, Colloq. Intern. Centre Natl. Rech. Sci. Paris, I19 (1963) I6r.

24 H. de Klerk, Govindjee, M. Kamen and J. Lavorei, Proc. Natl. Acad. Sci. U.S., 62 (I969) 972.

25 L. N. M. Duysens AND H. E. Sweers, in Studies on Microalgae and Photosynthetic Bacteria, Japan. Soc. Plant Physiol., University of Tokyo Press, Tokyo, I963, p. 353.

26 T. Yamashita And W. Butler, Plant Physiol., 44 (1969) 435.

27 J. Maroc, H. De Klerk and M. Kamen, Biochim. Biophys. Acta, r62 (I968) $62 \mathrm{I}$.

28 M. Ewald, Ph. D. Thesis, University of Paris at Orsay (rg69).

29 A. Krey and Govindjee, Proc. Natl. Acad. Sci. U.S., 52 (1964) I 568.

30 A. Krey and Govindjee, Biochim. Biophys. Acta, I 20 (1966) I.

3 I Govindjee, G. Papageorgiou and E. Rabinowitch, in G. G. Guilbault, Fluorescence Theory, Instrumentation and Practice, Marcel Dekker, New York, 1967, p. 5I I.

32 Govindjee, G. Döring and R. Govindjee, Biochim. Biophys. Acta, 205 (1970) 303.

Biochim. Biophys. Acta, 205 (1970) 36r-370 